\title{
Identifying neuropathic pain in patients with head and neck cancer: use of the Leeds Assessment of Neuropathic Symptoms and Signs Scale
}

\author{
Jean Potter BSc MRCP Irene J Higginson PhD FFPHM John W Scadding MD FRCP ${ }^{1}$ \\ Columba Quigley MD MRCP2
}

\section{SUMMARY}

The Leeds Assessment of Neuropathic Symptoms and Signs Scale (LANSS) is a simple bedside test in two parts-a patient-completed questionnaire and a brief clinical assessment. Its diagnostic capabilities have never been tested in patients with cancer pain. To determine these we conducted a prospective study in outpatients with head and neck cancer. All patients with pain completed the LANSS and underwent a medical assessment with a palliative care physician, whose findings were then reviewed by a pain specialist blinded to the LANSS scores. We assessed acceptability and understanding of the LANSS by patients and calculated the sensitivity and specificity of total LANSS scores and subscores derived from the patient-completed section.

Of 130 patients approached, 125 took part. $25(20 \%)$ of these had cancer related pain, mean score on an 11 point numerical rating scale 6.3 (median 6.0, range 3-10). Average age was 60 years (median 60, range 27-84); 56\% were male. LANSS completion time was about five minutes, and the procedure was acceptable to all patients. The pain specialist diagnosed neuropathic pain in 14/25 patients, in 13 of whom the neuropathic pain was part of a mixed pain picture. The LANSS correctly identified 11 of these cases (sensitivity $79 \%$; specificity $100 \%$ ). The patient-completed section alone had a sensitivity of $86 \%$ and a specificity of $91 \%$.

The LANSS is a simple and suitable screening test for neuropathic pain in patients with head and neck cancer related pain, although some modifications might improve it.

\section{INTRODUCTION}

Neuropathic pain is pain initiated or caused by a primary lesion or dysfunction of the nervous system. ${ }^{1}$ The distinction of neuropathic pain from other pathophysiological types of pain is fundamental in the management of patients with cancer because of the implications for investigation and treatment. ${ }^{2-4}$ Conventionally, the diagnosis of neuropathic pain relies on a detailed pain assessment conducted by a pain specialist. The 'gold standard' diagnostic test is therefore individual expert opinion. This method is labour-intensive and, since case definition may vary between experts, hinders comparisons between studies.

Department of Palliative Care and Policy, GKT School of Medicine, King's College London; ${ }^{1}$ National Hospital for Neurology and Neurosurgery; ${ }^{2}$ Hammersmith Hospitals NHS Trust, London, UK

Correspondence to: Dr Jean Potter, Department of Palliative Care and Policy, GKT School of Medicine, King's College London, Weston Education Centre, Cutcombe Road, London SE5 9RJ, UK

E-mail: jean.potter@kcl.ac.uk
The Leeds Assessment of Neuropathic Symptoms and Signs Scale (LANSS) is a standardized bedside test to identify patients in whom neuropathic pain mechanisms predominate. ${ }^{5}$ This tool has two parts-a patientcompleted section and a brief physical assessment. Five questions in the patient-completed section (maximum score 16) identify those who are experiencing phenomena associated with neuropathic pain: 'pins and needles' (paraesthesia); 'red skin' (autonomic changes); 'sensitive skin' (evoked dysaesthesia); 'electric shock pain'; and 'burning pain' (spontaneous dysaesthesia). The physical assessment (maximum score 8) is designed to identify allodynia by stroking cotton wool over the painful and the anatomically equivalent non-painful area, and altered pinprick threshold (PPT) by use of a 23 gauge needle to assess perception of pinprick in the same areas. The LANSS identifies patients with neuropathic pain by combining the scores of a patient's verbal description of pain and the results of neurological examination. A cut-off score of 12 points or more (out of a total of 24), when compared with expert opinion, had a sensitivity of $83 \%$ and a specificity of $87 \% .^{5}$ 
Pain affects $40-90 \%$ of patients with malignant disease, ${ }^{6}$ and neuropathic pain occurs in about one-third of patients with cancer-related pain. ${ }^{7}$ It is usually a mixed nociceptiveneuropathic pain phenomenon. ${ }^{6,8}$ The LANSS tool was developed and validated by assessing patients with pure neuropathic pain of largely non-malignant origin. A simple and quick test to identify patients with neuropathic pain in the general oncology clinic would be an important first step in improved pain control. However, the diagnostic capabilities of the LANSS in cancer-related mixedpathophysiology pain is unknown.

Although only around 5\% of all cancers arise in the head and neck, ${ }^{9}$ their highly erosive nature, the rich innervation of the head and neck and the intensive treatment regimens instigated mean that they are a common cause of pain,, $10-11$ and neuropathic pain in particular. ${ }^{7,12-13}$ Between $25 \%$ and $60 \%$ of patients with pain from head and neck cancer experience neuropathic pain. ${ }^{7,12-14}$

We therefore aimed to assess the acceptability and understanding of the LANSS by patients with head and neck cancer, and to calculate the sensitivity and specificity of the LANSS by comparison with medical expert opinion in these patients. In busy clinics a screening tool may be most useful when unaided patients can complete all parts; therefore the patient-completed subscores were also analysed separately to calculate a cut-off score with the highest sensitivity and specificity.

\section{METHODS}

\section{Design and patients}

Patients attending the head and neck cancer outpatient clinic at a London hospital were approached by a palliative care doctor (JP) in the clinic waiting area or by telephone survey. Potentially eligible patients were screened with a standardized format that explained the study and asked a screening question based on the first question of the Brief Pain Inventory. ${ }^{15}$ Those who experienced pain 'more severe or troublesome than everyday aches and pains' completed the LANSS and underwent a clinical assessment with the investigator (JP).

Patients were included in the study if they were over the age of 18 , were able to understand and speak English, and had head and neck cancer. Those patients with head and neck cancer with small-volume disease (staged at T1NOM0) and those who received only minor treatment (local excision and/or brachytherapy) were excluded.

The study was approved by the local hospital research ethics committee. Written informed consent was obtained from all patients.
Table 1 Demographic and medical characteristics of patients

\begin{tabular}{lc}
\hline Characteristic & $\begin{array}{l}\text { Head and neck } \\
\text { cancer patients } \\
\text { with pain (n=25) }\end{array}$ \\
\hline Mean age in years (range) & $60(27-84)$ \\
Male & 14 \\
Caucasian & 20 \\
Primary site & \\
Floor of mouth & 5 \\
Larynx & 4 \\
Tonsil & 3 \\
Tongue & 5 \\
Unknown & 4 \\
Other & 3 \\
Cancer treatment & \\
Radiotherapy & 21 \\
Chemotherapy & 2 \\
Surgery & 19 \\
Current stage of disease & \\
No evidence of recurrence & 24 \\
Metastases & 0 \\
Locoregional & 1 \\
\hline
\end{tabular}

${ }^{*}$ Most patients received multimodality treatment

\section{Measures and procedures}

A single investigator, JP, collected all data. Demographic and medical data (regarding disease severity, sequelae and treatments) were collected on all surveyed patients from their medical notes, by use of standardized proformas.

Initially all patients who agreed to take part completed the LANSS questions unaided. The investigator then reviewed the questions with each patient to verify patient understanding. If this review revealed questions that had initially been misunderstood by the patient, then the investigator clarified the answer with the patient and scores were amended accordingly. Where the question could not be answered by the patient either alone or in conjunction with the doctor, then the default answer was no (no score). Again this led in some cases to amended LANSS scores.

The investigator, using a standardized format, took a medical history and conducted a neurological examination including the sensory assessment component of the LANSS. Sensation (fine touch, pinprick and allodynia), power and appropriate tendon reflexes were assessed in all patients.

A pain specialist (JWS) who was blinded to the LANSS scores reviewed medical details of each case (demographic and medical data plus clinical assessment findings). The expert opinion of this pain specialist was used as the gold standard for identifying neuropathic pain in these patients. 


\section{Analysis}

Acceptability to patients was expressed as a percentage:

No. of patients who completed the LANSS

No. of patients who were invited to complete the LANSS

The sensitivity and specificity of the LANSS in these patients, compared with expert opinion, were calculated by use of the cut-off score of 12 suggested in the original validation study. ${ }^{5}$ The individual components of the LANSS were assessed by $\chi^{2}$ tests to identify which components were chosen more often by patients with neuropathic pain than those with non-neuropathic pain. Fisher's exact test was used to assess any association between two categorical variables when expected counts calculated for $\chi^{2}$ were below 5. The LANSS patient-completed section alone was analysed to calculate a cut-off score with the highest sensitivity and specificity.

Analysis was conducted with the Statistical Package for the Social Sciences version 10.1.

\section{RESULTS}

Of 130 patients who were approached, 125 took part. 26 $(21 \%)$ of these had had pain in the previous 24 hours that was 'more severe than everyday aches and pains'. One patient was found to have pain that was unrelated to the cancer and was excluded from the analysis. The mean score on an 11-point numerical rating scale for cancer-related pain was 6.3 (median 6.0; range 3-10). Table 1 shows the patients' demographic and medical characteristics.

\section{Acceptability and understanding by patients}

The LANSS was completed by all patients who were invited to do so. Completion time was about five minutes, including the sensory assessment. The full assessment could be conducted with the patient fully clothed in most cases. 6 patients $(24 \%)$ required help with the patient-completed section. Nine answers were amended on review of these 6 patients. In total, 25 patients answered five questions each. Amendment was necessary in 11 patients (9\%) (Table 2).

\section{Diagnosis of neuropathic pain}

Expert opinion diagnosed neuropathic pain in 14/25 patients (56\%). In 13/14 cases neuropathic pain was part of a mixed pain picture. Each pain quality assessed by the LANSS was experienced more often by patients with neuropathic pain than by those with non-neuropathic pain, but this reached statistical significance only for items related

Table 2 LANSS patient-completed section: difficulties with understanding

LANSS question

Question 1: Pins and needles

Does your pain feel like strange unpleasant sensations in your skin? Words like prickling, tingling and pins and needles might describe these sensations

Question 2: Red skin

Does your pain make the skin in the painful area look different from normal? Words like mottled or looking more red or pink might describe this appearance

Question 3: Sensitive skin

Does your pain make the affected skin abnormally sensitive to touch? Getting unpleasant sensations when lightly stroking the skin or getting pain when wearing light clothes might describe this abnormal sensitivity

Question 4: Electric shock pain

Does your pain come on suddenly and in bursts for no reason when you are still? Words like electric shock, jumping and bursting describe these sensations

Question 5: Burning pain

Does your pain feel as if the skin temperature in the painful area has changed abnormally? Words like hot or burning describe these sensations
Frequency and reasons for amendment of initial answer

Amendments $=0$

Amendments $=3$

Difficulty distinguishing between long-term skin changes due to treatment and intermittent skin changes occurring with pain $(n=2)$ Difficulty visualizing painful area $(n=1)$

Amendments $=3$

Pain experienced only when carrying heavy bags over affected shoulder $(n=2)$

Pain experienced only affecting the mucous membranes of the mouth $(n=1)$

Amendments $=3$

Pain experienced only on movement $(n=2)$

Pain experienced only on swallowing cold drinks $(n=1)$

Amendments $=2$

Pain experienced only on swallowing warm drinks $(n=2)$ 
Table 3 Presence of pain qualities according to underlying pathophysiology

\begin{tabular}{llll}
\hline Pain quality & $\begin{array}{l}\text { Neuropathic } \\
\text { pain }(\mathbf{n = 1 4}\end{array}$ & $\begin{array}{l}\text { Non-neuropathic } \\
\text { pain }(\mathbf{n = 1 1}\end{array}$ & Significance \\
\hline Pins and needles & 6 & 4 & $\chi 0.11 P=0.53^{\star}$ \\
Red skin & 7 & 2 & $\chi 2.71 P=0.11^{\dagger}$ \\
Sensitive skin & 12 & 1 & $\chi 14.5 P=0.0001^{*}$ \\
Electric shock pain & 9 & 2 & $\chi 5.3 P=0.03^{*}$ \\
Burning pain & 7 & 3 & $\chi 1.33 P=0.23^{\dagger}$ \\
Allodynia & 12 & 0 & $\chi 18.1 P=0.0001^{*}$ \\
Altered pin prick threshold & 13 & 0 & $\chi 21.3 P=0.0001^{*}$ \\
\hline
\end{tabular}

${ }^{*} \chi^{2}$; ${ }^{\dagger}$ Fisher's exact test

to 'sensitive skin', 'electric shock pain', allodynia and altered PPT (Table 3).

With the suggested cut-off score of 12, the LANSS correctly identified $11 / 14$ cases of neuropathic pain (sensitivity 79\%; specificity 100\%). Amendment of responses changed the total LANSS scores of 6 patients, but this had no effect on the sensitivity or specificity.

A cut-off score of 6 or more, with data from the unaided patient-completed section, gave sensitivity and specificity scores of $86 \%$ and $82 \%$, respectively. When the amended patient-completed scores were used the sensitivity was unchanged but the specificity rose to $91 \%$.

\section{DISCUSSION}

This study indicates that the LANSS is a valid test for identifying neuropathic pain in patients with pain from head and neck cancer. A total score of 12 or more had a sensitivity of $79 \%$ and a specificity of $100 \%$.

Some of the LANSS questions were difficult for patients to complete accurately. For example, skin redness was associated with either pain or treatment. Patients with damaged mucous membranes experienced sensitive skin and burning sensations but these were not, on medical assessment, related to neuropathic pain mechanisms. Despite these drawbacks, a cut-off score of 6 (maximum 16) on the patient-completed subscore alone had a sensitivity and specificity of $86 \%$ and $82 \%$, respectively. Amendment of patients' answers by giving no score to questions regarding sensitive skin and burning sensations in patients with damaged mucous membranes, and by asking whether skin colour change occurred only when pain was particularly severe, had no effect on the sensitivity but improved the specificity.

In this study, 1 in 5 patients attending a head and neck outpatient clinic had pain, and over half of these had by others. $2,7,12-14,16-19$ The total LANSS score correctly identified $86 \%$ of these patients, and the patient-completed data offered the same sensitivity with only slight loss of specificity. Identification of patients with neuropathic pain is an important first step towards control of this difficult symptom. A shortened version of the LANSS, comprising a modified patient-completed section alone, could be used as a screening tool for neuropathic pain in the waiting area of busy oncology clinics.

Acknowledgment The study was supported by the Mrs Coco Markus Clinical Research Fellowship.

\section{REFERENCES}

1 Merskey H, Bugduk N. Classification of Chronic Pain. Descriptions of Chronic Pain Syndromes and Definitions of Pain Terms. Seattle: IASP Press, 1994

2 Robertson MS, Hornibrook J. The presenting symptoms of head and neck cancer. N Z Med J 1982;95:337-41

3 Twycross R. Symptom Management in Advanced Cancer. Oxford: Radcliffe Medical Press, 1997

4 Twycross R. Cancer pain classification. Acta Anaesthesiol Scand 1997;41:141-5

5 Bennett M. The LANSS Pain Scale: the Leeds assessment of neuropathic symptoms and signs. Pain 2001;92:147-57

6 Portenoy RK. Cancer pain. Epidemiology and syndromes. Neurologic Clinics 1993;63:2298-307

7 Grond S, Zech D, Diefenbach C, Radbruch L, Lehmann KA. Assessment of cancer pain: a prospective evaluation in 2266 cancer patients referred to a pain service. Pain 1996;64:107-14

8 Caraceni A. Clinicopathologic correlates of common cancer pain syndromes. Haematol/Oncol Clin N Am 1996;10:57-78

9 Sanderson RJ, Ironside JAD. Squamous cell carcinomas of the head and neck. BMJ 2002;325:822-7

10 Caraceni A. An international survey of cancer pain characteristics and syndromes. Pain 1996;82:263-74

11 Brescia FJ, Adler D, Gray G, Ryam MA, Cimino J, Marntani R. Hospitalised advanced cancer patients: a profile. J Pain Symptom Management 1990;5:221-7 
12 Chua KSG, Reddy SK, Lee M, Patt RP. Pain and loss of function in head and neck cancer survivors. J Pain Symptom Management 1999; 18:193-202

13 Vecht CJ, Hoff AM, Kansen PJ, de Boer MF, Bosch DA. Types and causes of pain in cancer of the head and neck. Cancer 1992;70:178-84

14 Grond S, Zech D, Lynch J, Diefenbach C, Schug SA, Lehmann KA. Validation of the World Health Organisation guidelines for pain relief in head and neck cancer. Ann Otolaryngol Rhinol 1993;102:342-8

15 Daut RL, Cleeland CS, Flanery R. Development of the Wisconsin Brief Pain Questionnaire to assess pain in cancer and other diseases. Pain 1983; 17:197-210
16 Greenslade R, Portenoy RK. Pain syndromes in head and neck cancer. $J$ Pain Symptom Management 1988;3(suppl):S21

17 Keefe FJMGBA, Crisson J. Pain in the head and neck cancer patient: changes over treatment. Head Neck Surg 1986;8: $169-76$

18 Chaplin JM, Morton RP. A prospective, longitudinal study of pain in head and neck cancer patients. Head Neck 1999;21: 531-7

19 Aird DW, Bihari J, Smith C. Clinical problems in the continuing care of head and neck cancer patients. Ear Nose Throat J 1983;62: 30 